\title{
Spitzer Mission Operation System planning for IRAC Warm-Instrument Characterization
}

\author{
Joseph C. Hunt, Jr.* and Marc A. Sarrel ${ }^{\dagger}$ \\ Jet Propulsion Laboratory, California Institute of Technology, Pasadena, CA 91009 \\ and \\ William A. Mahoney, Ph.D. \\ California Institute of Technology, Pasadena, CA 91125
}

\begin{abstract}
This paper will describe how the Spitzer Mission Operations System planned and executed the characterization phase between Spitzer's cryogenic mission and its warm mission. To the largest extend possible, the execution of this phase was done with existing processing and procedures. The modifications that were made were in response to the differences of the characterization phase compared to normal phases before and after. The primary two categories of difference are: unknown date of execution due to uncertainty of knowledge of the date of helium depletion, and the short cycle time for data analysis and re-planning during execution. In addition, all of the planning and design had to be done in parallel with normal operations, and we had to transition smoothly back to normal operations following the transition. This paper will also describe the re-planning we had to do following an anomaly discovered in the first days after helium depletion.
\end{abstract}

\section{Introduction}

The Spitzer Space telescope was launched on 25 August 2003 with a prime Cryogenic Mission Requirement of five years. Following launch the In Orbit Checkout (IOC) and Science Verification (SV) mission phases were conducted to calibrate the instruments' and spacecraft's performance before routine science observations began. The helium depleted on 15 May 2009, ending the prime mission and starting the Infrared Array Camera (IRAC) Warm Instrument Characterization (IWIC) followed by the extended mission. The mission phases referred to herein are IOC/SV, the Prime Cryogenic, IWIC and Extended Warm. The Extended Warm Mission is approved for two years with a pending proposal for an additional two-year extension. The spacecraft team has identified several issues that may require changes to operations in the extended mission through the end of 2013.

\footnotetext{
* Mission Operations Engineer Team Lead, Spitzer Space Telescope, Jet Propulsion Laboratory, California Institute of Technology, M/S 264-767, 4800 Oak Grove Dr., Pasadena, CA 91109.

${ }^{\dagger}$ Mission Operations System Engineer, Spitzer Space Telescope, Jet Propulsion Laboratory, California Institute of Technology, M/S 264-767, 4800 Oak Grove Dr., Pasadena, CA 91109.

* Operations Team Lead, Spitzer Space Telescope, California Institute of Technology, M/S 3146, 1200 East California Blvd, Pasadena, CA 91125.
}

American Institute of Aeronautics and Astronautics 
Table 1, Mission Phases, is a list of dates and durations for each phase. The IWIC phase began once the project determined that the helium had run out, and the spacecraft was returned to its normal operating mode.

The purpose of IWIC was to characterize and calibrate the IRAC instrument at the warmer operating temperature. Following the depletion of the cryogen only the two shortest wavelength detectors of IRAC, $3.6 \mu \mathrm{m}$ and $4.5 \mu \mathrm{m}$, produce valid science data at the warmer, passive equilibrium temperature of the telescope. No changes in spacecraft performance

Table 1: Mission Phases

\begin{tabular}{|l|l|}
\hline Phase & Start and End Dates \\
\hline Launch & $2003-08-25$ \\
\hline IOC and SV & $2003-08-25 / 2003-12-01$ \\
\hline Prime Cryogenic & $2003-12-01 / 2009-05-15$ \\
\hline Planned Standby & $2009-05-15 / 2009-05-16$ \\
\hline IWIC Overall & $2009-05-16 / 2009-07-27$ \\
\hline IWIC Post-Anomaly Only & $2009-06-18 / 2009-07-27$ \\
\hline Extended Warm Approved & $2009-07-27 / 2011-12-31$ \\
\hline Extended Warm Proposed & $2012-01-01 / 2013-12-31$ \\
\hline
\end{tabular}
were expected at the warmer temperature, and none were observed during IWIC. Because no changes were expected, we planned and executed only routine spacecraft maintenance activities during IWIC.

We had a requirement to complete IWIC within six weeks of helium depletion, with a goal of four weeks. Almost immediately after the helium depletion an IRAC instrument anomaly occurred. This paper will describe how the re-planning was developed to accommodate the delay in the start of IWIC.

The main differences between IOC and IWIC were that (1) the level of staffing was much lower during IWIC compared to IOC, (2) in IOC we had nearly continuous communications with the observatory versus two four-hour sessions per day during IWIC, and (3) the scope of IWIC was much smaller than IOC.

In IOC special teams were established for the planning and execution of activities that were not part of the nominal operation phase. Three instruments had to be managed instead of one. Some of the IOC/SV activities were conducted with the observatory at a fixed attitude to enable constant communication. There were no limiting factors for ground communication resources because the telecom link margin was not an issue during IOC/SV. This differed in IWIC and required advance planning to manage the downlink bit rates. The scope of IWIC was much smaller because the only change expected was to the final temperature of the IRAC instrument and the array operating parameters.

The spacecraft performance has been demonstrated over the course of the five-year cryogenic mission. No new spacecraft functionality was required for the IWIC mission phase. All spacecraft trending data indicated that the spacecraft continued to perform as it had during the Cryogenic mission phase.

The Cryogenic mission was designed with science collection of three instruments: the Infrared Array Camera (IRAC), the Infrared Spectrograph (IRS), and the Multiband Imaging Photometer for Spitzer (MIPS). At the warmer temperature, the MIPS and IRS instruments will not return scientifically valid data and are not being operated as part of the Spitzer noncryogenic, warm, or extended mission.

The mission design for the Cryogenic Mission was optimized for science return based on the Mission Operating System (MOS) planning efficiency. The same high efficiency will not be required for the Warm Mission. 
The Spitzer mission as a whole is composed of the flight system, the ground system, the launch system, and systems used in assembly, verification and validation of the spacecraft. This paper will focus on the system engineering of the Spitzer Mission Operation System for the planning and execution of IWIC to the transition to the Warm Mission. The MOS is divided into the flight and ground systems. The flight system is composed of the spacecraft and the science instruments, which are each composed of hardware and software. The ground system is composed of the MOS and the Ground Data System (GDS). The MOS contains the people, teams, processes and procedures used to operate the mission. The GDS is made up of ground data system hardware and software. This includes not only computers and networks, but distributed physical facilities like the mission support areas, the science center and multi-mission facilities.

\section{Prime Mission Overview}

The Spitzer mission is the last of NASA's four great observatories. The first three were, in order of launch: Hubble Space Telescope in 1990, Compton Gamma Ray Observatory in 1991, and Chandra X-Ray Observatory in 1999. It is run, in many ways, like a ground-based observatory. There is a yearly call for proposals and anyone can submit a proposal for observation time. Spitzer was launched on August 25, 2003. The in-orbit checkout ended December 1, 2003. The primary operations phase lasted from then until the helium depleted, May 15, 2009.

In 1998, the science objectives for the Spitzer mission were defined as:

- Deep surveys of oldest galaxies

- Evolution and structure of ultra-luminous galaxies and quasars

- Search for Brown Dwarfs

- Evolution of stellar disks and planetary systems

For the extended warm, IRAC-only mission, the new science objectives are:

- Study properties of extra-solar planets

- Study galaxies during the first one billion years after the Big Bang

- Complete census of the galaxy for young stars

- Determine cosmic distance scale in the local universe

The primary mirror is $85 \mathrm{~cm}$ in diameter. It is made of beryllium and was cooled to between about $5.6 \mathrm{~K}$ and $12 \mathrm{~K}$, depending on the instrument in use. The field-of-view angle of the telescope is $32^{\prime}$ ( 32 arcminutes). The focal length is $10.2 \mathrm{~m}$. The total mass of the spacecraft at launch was $950 \mathrm{~kg}$, including $50.4 \mathrm{~kg}$ of liquid helium cryogen.

The spacecraft uses its on-board pointing control system to shade itself with its solar arrays for reasons of thermal control. As such, at any given time, it can see only about $31.5 \%$ of the full sky (Figure 1). This area is called the Operational Pointing Zone (OPZ). There are two zones of the sky around the ecliptic poles that are always in view. Objects in the ecliptic plane are in view for two periods of forty days each per year.

Spitzer is in a heliocentric, Earth-trailing orbit. It follows the Earth around the Sun. Its orbit is slightly more elliptical than the Earth's, and most of the time it is farther away from the Sun than the Earth is, so it slowly recedes from Earth at about $0.1 \mathrm{AU} / \mathrm{yr}$. This recession decreases the telecommunications performance and causes the use of larger ground antennas, or arrays, to maintain the desired downlink bit rate. At the time the helium was exhausted, the one-way light time from the Earth to Spitzer was about twelve minutes. During the cryogenic mission there was a telecommunication session, about one hour in length, with the spacecraft approximately 
every twelve or twenty-four hours. The time between telecom sessions, where science data are collected, is called a Period of Autonomous Operations (PAO).

Because of thermal and power constraints, only one of the three science instruments could be powered on at one time. Each such period was called a campaign and usually lasted from about one to three weeks. The dates and durations of campaigns were determined by the number, type and distribution on the celestial sphere of the approved science targets and on their observing constraints.

Activities on the spacecraft are controlled by sequences of commands. Independent of campaign boundaries, each command sequence executes on the spacecraft for approximately one week. The development of each sequence begins approximately five or six weeks before the start of its execution. During the first three

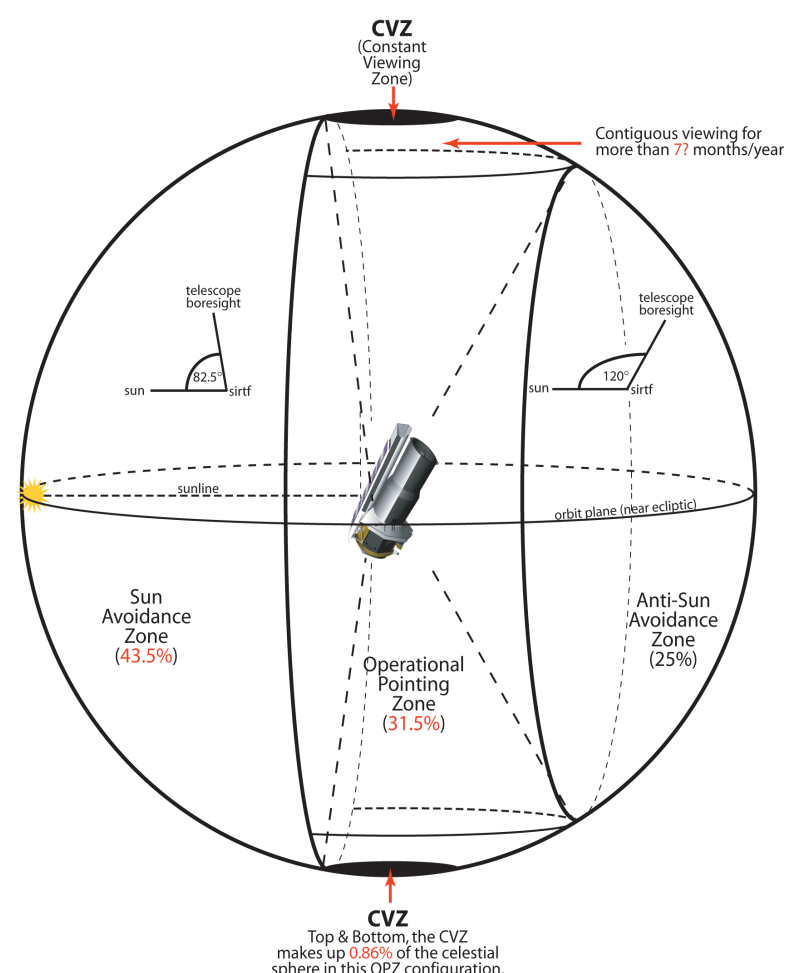

Figure 1. Spitzer's Operational Pointing Zone weeks of sequence development, the sequencing teams schedule the observations, instrument calibrations, spacecraft calibrations and telecommunication sessions. During the next two weeks of sequence development, they translate that schedule into a sequence of events that the spacecraft can execute, and check that the sequence does not violate any rules. This period in the development cycle includes enough time to correct errors in the sequence.

The processing of science data on the ground is highly automated. The images are processed following each telecommunication session. The science data packets from the spacecraft are transformed into un-calibrated images at JPL and delivered to the Spitzer Science Center (SSC). Once at the SSC, they are calibrated and merged into higher-level data products, analyzed for data quality, distributed to the observer, and archived.

\section{A. Driving Requirements and Mission Constraints for IWIC}

The driving requirements for IWIC were structured to establish the baseline functionality for collecting science with the IRAC instrument operating at approximately $30 \mathrm{~K}$. Constraints to the IWIC sequencing were imposed to support the boundaries of development and execution. The below list is an example of some of the requirements and constraints:

1. IWIC driving requirements

- Verify that all observatory subsystems are nominal,

- Verify that warm IRAC can take data,

- Set the operating parameters in the data taking blocks to support nominal operation of the warm IRAC,

- Determine that the data taken with the warm instrument, after deployment of the updated sci2 block library, can be calibrated. 


\section{Mission constraints}

- 2 work shifts,

- Shifts would cover 7 days per week,

- Telecommunications passes ( 1 every 12 hours) unlike IOC continuous coverage,

- Relative timed sequences due to the date of helium depletion being unknown,

- Each sequence was built to a fixed length that could later be trimmed to fit telecommunications allocations,

- Avoid bright objects during the idle time,

- Shorter sequences allowed rapid turn-around for data analysis for sequence dependencies,

- While still building Prime mission Cryo sequence start to pre-build IWIC sequences.

\section{B. Transitions between Mission Phases for IWIC}

This section defines the spacecraft and ground system state at three times during the transition from prime cryogenic mission to the nominal warm mission. The operations ground system remained in its normal, prime-mission configuration throughout all the mission phases with the exception of updated versions of the red-alarm limits file, and the ground changes to use the new sci2 block library for sequence development.

The mission phases that were associated with IWIC are:

- The transition from the prime cryogenic mission to helium depletion (Standby Mode) anomaly recovery,

- The transition from (Standby Mode) anomaly recovery to IWIC,

- The transition from IWIC to the extended warm mission.

Table 2. Margin Within Mission Phase

\begin{tabular}{|l|l|l|l|l|}
\hline Mission Phase & $\begin{array}{l}\text { Prime } \\
\text { Cryogenic }\end{array}$ & $\begin{array}{l}\text { Planned } \\
\text { Standby Mode }\end{array}$ & IWIC & Extended Warm \\
\hline $\begin{array}{l}\text { Expected Total } \\
\text { Duration }\end{array}$ & 5 years & 3 days & 42 days & 2 years \\
\hline $\begin{array}{l}\text { Margin within } \\
\text { Total }\end{array}$ & $\mathrm{n} / \mathrm{a}$ & 1 day $(33 \%)$ & 8.4 days $(20 \%)$ & n/a \\
\hline
\end{tabular}

1. Prime cryogenic Mission to Standby Mode

The spacecraft was in its normal, prime-mission configuration. No changes to the spacecraft configuration were made to support IWIC or the warm mission before the helium depleted. At the time of helium depletion:

- All instruments and the combined electronics were turned off, the combined electronics is not needed for IRAC,

- All sequences are unloaded,

- The spacecraft remain in an Earth point fixed attitude.

2. Standby Mode to IWIC

During the anomaly recovery mode, the anomaly response team made the determination that the spacecraft had indeed run out of helium. The criterion for helium depletion was that the cryogen tank reached a temperature of $2.18 \mathrm{~K}$. At this temperature, super-fluid helium transitions to normal fluid. At the end of the standby-mode recovery, the Anomaly Response Team returned the spacecraft to the following configuration: 
- The spacecraft to nominal mode with the high-gain antenna at Earth point,

- IRAC was powered on as part of the first IWIC mini sequence,

- The combined electronics were turned off,

- A dummy master sequence was loaded and activated,

- The updated version of the sci2 block library with the no-op commands to support the first portion of IWIC.

\section{IWIC to Nominal Warm Mission}

Before the end of IWIC, the project conducted an internal IWIC exit review. The review confirmed that the project has met all the requirements and objectives before the start of the warm mission science.

At the completion of the IWIC, the MOS teams placed the spacecraft in the following configuration.

- The updated version of the sci2 block library with the final parameter values to support IRAC warm mission operations were loaded and activated,

- The last IWIC sequence was completed,

- The first warm mission sequence was loaded on board and activated,

- The fault-protection response was changed to always set the make-up heater to $0 \mathrm{~mW}$. The make-up heater is no longer needed in the warm mission.

\section{IWIC Objectives}

The IRAC warm instrument characterization consisted of a set of on-orbit tests and observations with five primary objectives: First, to check the functionality of IRAC at $\sim 30 \mathrm{~K}$, second, to determine the best operating parameters (array biases, temperature set points, observation frame times and Fowler numbers, used to reduce the read noise), third, to characterize properties of the data such as noise and artifacts, fourth, to collect calibration data to determine the dark bias, flat-field, linearity and other required calibrations to convert the data from raw data number to flux-calibrated images in units of milliJansky per steradian (mJy/sr), and fifth, to perform representative science observations to inform the observing community of the capabilities of warm IRAC.

\section{IWIC Development Phase}

The planning process for the IWIC was conducted by the Mission Operations System (MOS), through the forum of the Mission Operations System Design Team (MOSDT) meetings. Some engineering decisions that supported the development of this plan were made at Flight Engineering Team (FET) meetings. At two different times during the development phase, we conducted table-top design reviews. These table-top meetings were a design exercise to walk through the MOS plan and detailed schedule for IWIC. All MOS teams participated, and the table-tops proved to be useful and helped us identify areas for improvement in the plan.

In December 2008, we published an IWIC Plan that consolidated the MOS planning into a formal document. A formal review was conducted in January 2009, and gave full concurrence to proceed with the plan as written. A series of schedules were developed to layout the work needed to plan and execute the IWIC.

The original IWIC plan design goals were to be as responsive as possible to late changes from the IRAC team during IWIC sequence execution. For implementation of this, the MOS design required:

- Sequence length of 12 hours vs. the nominal 7 days, 
- Short lead time for building sequences 2 to 3 days,

- For unique cases the sequence development cycle was less than 2 days,

- Teams worked two shifts per day (prime and second),

- Build the first Nominal Warm mission sequences on a compressed schedule during IWIC,

- Mini sequences were built to execute in relative time vs. absolute time.

To the maximum extent practical, the IWIC planning included detailed timeline walkthroughs and a pre-build, review, and approval of all mini sequences as they were expected to be implemented because the actual performance of the observatory and instrument at higher temperatures was not yet known. The walkthroughs were a series of table top exercises designed to validate the transition between the anomaly response, re-planning to pre-build mini sequences development after the exact date of helium was determined and sequencing for the transition to the nominal warm mission. Implementation and schedule drivers were known to include uncertain telecommunications allocations, concern about latent images on the arrays, and block library updates

The sequence products for the majority of activities for the IWIC were built and tested in advance because we wanted to validate the build process and minimize the workload during IWIC. The only types of work we anticipated during IWIC were changing the mini sequence length to fit the telecommunications allocation, changing targets because the original targets may no longer be in the OPZ, and changing the setting for the IRAC instrument based on data results from previous mini sequence execution.

There was a concern that the compressed timeline to build the first few warm-mission sequences might lead to a lower observational efficiency. Normally a significant portion of the sequence development time is spent minimizing the duration of spacecraft slews in order to allow more science observations. However, by selecting programs that were relatively easy to schedule (e.g. minimal or no observational constraints) and which placed minimal demands on the instrument performance, efficiency remained high.

During IWIC execution, the MOS teams had to start building sequencing for the nominal warm mission in parallel with the development of IWIC sequences. Many of the people who worked on the IWIC were also required to build the nominal science sequences for the warm mission. The project discussed several approaches to minimize this anticipated spike in workload. The optimum was to do as much work as is reasonably possible in advance to minimize the height of the peak workload once the helium was exhausted.

The science observations planned for the first year of warm operations were approved by end of December 2009. They were then staged in the science operations data base so that they could be scheduled. The first phase in the sequence building process is to place in the timeline the telecommunication allocations and the spacecraft engineering activities and science calibrations. Because most of these activities are implemented on routine bases, the MOS was able to perform work in advance to save effort when the helium is depleted

During the month-long IWIC, some MOS and SSC teams were required to work extended shifts, generally two shifts per day, including weekends. Based on the telecommunications allocations, the Flight Control Team (FCT) was available 24/7. The shift start and end times changed in coordination with the telecommunications allocations (one downlink every twelve or twenty-four hours). The IRAC instrument support team (IST) often worked three shifts per day, especially during the anomaly recovery, and during the first two weeks of IWIC. At the end of IWIC, the teams began to transition to their planned warm mission staffing profiles. 
The IWIC operational organization is the same as that for nominal operations with three additions:

1. IWIC Lead - The IWIC lead and deputy were responsible for coordinating the activities of the MOS across teams during the IWIC. The IWIC lead tracks progress compared to the master IWIC schedule. The IWIC lead did not assume the responsibilities of the Mission Manager.

2. Focus Adjust Team - The purpose of the Focus Adjust Team was to adjust the focus of the telescope in the anomalous case where the focus is found to be out of specification.

3. Frame Table Update Team - There were two purposes for the Frame Table Update team. The first was to perform an update to the frame table, if necessary, for IRAC highprecision photometry following the conclusion of IWIC. The second was to update the frame table during IWIC in the anomalous case where the frame table is found to be out of specification. Frame tables are used to specify the alignment between the spacecraft, telescope and instrument coordinate systems.

Neither the focus adjust or the frame table update was planned as part of the IWIC baseline schedule. If either had to be adjusted it would be treated as an anomaly and the planned IWIC activities would be put on hold. In fact, neither a focus adjustment nor a frame table update was required.

\section{Planning Process Description}

The overall strategy for implementing the mini sequences was similar to the style of sequencing during IOC. Sequencing with the mini sequences allows more flexibility in the packaging of activities. There were two major drivers for the planning process.

The first driver was to minimize the risk of latent images from bright sources. Aside from the final temperature of the arrays, the potential effect from latent images was probably the biggest unknown and one of significant concern. Of course none of the latent images that plagued the cryogenic mission were present because the arrays were operating at temperatures higher than the cryogenic anneal temperature. However, there had been a concern that other charge collection effects could cause serious latent images, possibly permanent ones, which would significantly change the IRAC observing strategy and performance for the warm mission. Thus the IWIC sequences were planned with great attention paid to either avoiding bright sources or to minimize the time spent pointing at them. Since bright sources could be in the field of view during downlinks, our strategy was to minimize the time spent at Earth-point. As it turned out, latent images from bright sources are greatly reduced from those experienced during the cryogenic mission.

Our second goal was to develop fifty-three pre-build sequences with maximum likelihood of being flyable with minimal or no changes. Throughout IWIC, the telecommunications allocations provided two tracks per day, each with a four-hour duration but with a somewhat non-uniform spacing. Since the time between station passes varied from a minimum of about eight hours to a maximum of about sixteen hours, we built sequences that included about eight hours of IWIC activities followed by about eight hours of short observations that took the observatory to a dark region in the constant viewing zone.

In order to facilitate their prioritization, the order and duration of the actual IRAC activities was determined through months of planning by the IRAC team based on a logical and conservative flow. This involved first powering on IRAC at the higher temperatures and assessing the basic operation. This step was always considered the most risky. While the 
telescope was still warming, the performance was measured over a range of detector operating parameters, including array temperatures and bias settings. Once the final operating temperature was determined, detailed checkouts and calibrations were performed. In addition to these sequences, two new versions of the science block library were required. The first, which was implemented before the start of IWIC, replaced the array temperature and bias commands with no-op commands, leaving control of these parameters to the Instrument Engineering Requests (IERs) built into the sequences. The second block library, implemented about two weeks into IWIC, set the commands to the new operating temperature and array settings. The workflow, activity durations, and staffing requirements were modified and then validated through several tabletop sessions and dry runs.

To make sure we had sufficient staffing for IWIC, the IRAC IST at the SSC worked in close collaboration with the IRAC Instrument Team at the Smithsonian Astrophysical Observatory (SAO). The IST staffing during IWIC reached 8 Full-Time Equivalents (FTEs), about 3 above the cryogenic staffing level with the added personnel being internal SSC members familiar with the operation of IRAC. Two members of the IST were assigned to scheduling within the IST, and the remaining members assigned as principals for the IWIC tasks. SAO contributed 2 FTEs. The Observatory Planning and Scheduling Team (OPST) staffing remained constant at 4.5 FTEs. For both teams and for the duration of IWIC, coverage was planned for sixteen hours per day, seven days a week. The product development for the pre-built sequences was carried out with existing staff and done in parallel with the normal cryogenic sequence development.

As a result of the temperature anomaly during IWIC, the content of individual mini-sequences was rearranged significantly, although many of the basic activities remained valid.

Most of the planned IWIC activities were put on hold while the IRAC software patch was being developed and implemented. However, several tests on the observatory were conducted to verify the cause of the anomaly and to confirm the fix would work.

While the IRAC array temperature control software patch was being developed, considerable observatory time was not needed for IWIC activities. A very distant gamma-ray burst (GRB) had been detected on 23 April 2009 and several long sequences were built to observe it with the array temperature either controlled, when possible, or left floating upward very slowly. This was an ideal science investigation because the sequences were easy to build and placed minimal demand on the observatory. They also kept the observatory pointing to a safe and dark region of the sky. More complicated science was precluded because the IRAC team was consumed with recovery from the anomaly and re-planning IWIC.

The initial plan called for two sequences per day with roughly 8 hours of IWIC activities in each. Following the anomaly, most sequences were extended to about 24 hours, and most had little or no padding. This both increased the efficiency and reduced the sequencing effort. For OPST and subsequent teams, the effort scaled roughly as the number of sequences, rather than as their duration. Thus the number of sequences was cut by nearly a factor of two.

Because of the very slow warm up, the final array temperature was not known until about two months after the beginning of normal warm mission operations.

\section{Sequence Process Description}

We used several different types of sequence products during IWIC and the beginning of the warm extended mission. All of the products related to IWIC itself were built in advance, to the highest level of maturity possible. The absolute-timed sequences for the warm extended mission could not be pre-built, and were built completely during the latter part of IWIC. During IWIC, 
we built (a) relative-timed mini sequences, (b) an update to a block library, (c) a weeklong sequence for the final portion of IWIC, and (d) the first several sequences for the warm, extended mission.

We built fifty-three mini sequences several months in advance of IWIC. Each mini sequence was sixteen hours long. It contained eight hours of real IWIC activities, and eight hours of safe, idle observations to reduce the probability of leaving latent images on the IRAC arrays.

During IWIC itself, each mini sequence was trimmed by removing some of the idle observations to make the total duration fit between our scheduled telecommunication sessions. The telecommunication sessions were scheduled roughly twelve hours apart, plus or minus four hours. Because we did not know the data of helium depletion in advance, we did not know on which day each mini sequence would be executed and hence, did not know the correct length to make it fit between the telecommunications sessions. We also built several extra mini sequences that consisted exclusively of the safe, idle observations. These were built as a contingency in case we were to fall behind our sequence development schedule and could not uplink our planned mini sequence.

In addition to trimming, mini sequences were changed to add, remove or modify IWIC activities. These changes were done in response to either the results of prior IWIC mini sequences, or because some details of the activities could not be known in advance.

In the original plan, we allotted four days to the baseline cycle for mini sequence development, execution and analysis with one pair of twelve-hour sequences starting each day. Using this pattern, for example, the first mini sequences that could be modified based on the results of mini sequence Q or R (started on day nine), would be mini sequence $\mathrm{Y}$ or $\mathrm{Z}$ (started on day thirteen). In advance of IWIC, we identified several cases where the four-day cycle time was too long to accommodate results of one sequence into another. So, we came up with a compressed three-day timeline. Sometimes, the beginning of the cycle was compressed, other times, the end. This allowed results from mini sequence $\mathrm{C}$, started on day two to be incorporated into mini sequence $\mathrm{G}$, started on day five. To accommodate this, as an exception to our two-shift rule, we scheduled some of the compressed work to be done on third shift. In one case a single mini sequence was compressed on both ends, resulting in a two-day cycle time rather than the baseline four-day.

Normally, key operating parameters for the IRAC instrument are stored on-board the spacecraft in what is named the sci2 block library. One of the primary goals of the first week of IWIC was to determine the optimum new values for those parameters for the warm extended mission. Subroutines from this block library are called before each observation to set the parameter values. Prior to IWIC, a new version of the sci 2 block library was built that contained no-op commands instead of commands to set the values of the parameters. That no-op version was activated on the spacecraft before the beginning of the first mini sequence. The mini sequences were built to set the values of the parameters themselves. This allowed the parameters to be varied from observation to observation to help find their optimal values. Once those values were determined, a new version of the block library was built, tested on the ground and sent to the spacecraft. That whole process was scheduled to take four days from when the final values of the parameters were determined, until the new version of the block library was activated. Once the block library was active on the spacecraft, the need for IERs to set the values was eliminated. The only constraint was that it had to be activated before the final, week-long, absolute-timed IWIC sequence was built on the ground. This was the transition to the first sequence of the extended warm mission. 
Because it was absolute-timed, this final IWIC sequence could not be pre-built. Nominally, it takes us thirty-seven days from the start of sequence building to the start of execution for a oneweek, absolute-timed sequence. For the final week-long sequence of IWIC, we did the same job in seventeen days. Much of the savings was because the sequence only contained IRAC instrument engineering activities rather than science observations. In order to build this last IWIC sequence, we had to predict when all the mini sequences would be completed, and choose a time for the start of warm, extended-mission sequences.

We had to start building the first warm, extended-mission sequences during IWIC as well. But, we could not begin that task until about two weeks into IWIC. The three prerequisites for the warm, extended-mission sequences were: (a) execution of most of the mini sequences, (b) development started on the sci2 block library, and (c) development started on the final weeklong IWIC sequence. That allowed us much less time than usual to build the warm, extendedmission sequences. Normally, our sequences begin executing on Wednesdays and run for seven days. We could not completely control the start date of the first warm sequence. So, we decided that it was only required to end on Wednesday, to get us back to our normal pattern. It could start on any other day of the week, and hence could be anywhere from about three to ten days in length.

To accommodate this, we planned to build the first five warm, extended-mission sequences in much less time than the nominal thirty-seven days. Each of the first five sequences was allowed a little more time than the preceding one: 20,21, 24, 28 and 32 days, until the sixth sequence was built in the normally allotted thirty-seven days. This is the one part of the plan where we fell behind schedule significantly. It actually took us until the thirteenth sequence to return to the normal build pattern. It took longer than planned because of the additional calibration required when we lowered the set point temperature after IWIC.

All of the above work had to be laid out in such a way that we could accommodate it within our staffing constraints. We also had to accommodate dependencies between products. The primary dependencies were (a) the choice of parameters to go in the block library, (b) the choice of time for the end of the mini sequences, (c) the end of the final week-long IWIC sequence, and (d) the beginning of the warm, extended mission.

\section{IWIC Anomaly, Re-planning Process and Execution}

We discovered the temperature-control anomaly immediately after the execution of the first IWIC mini sequence. We were unable to correctly control the temperature of the IRAC instrument, at the new warmer temperature, as we had done for more than five years during the prime cryogen mission. We halted the second IWIC mini sequence, which was then executing on board the observatory, and put IWIC execution on hold (by commanding the spacecraft in standby mode again) to allow ourselves time to resolve the anomaly. In some cases, the anomaly caused the instrument to be heated to temperatures higher than desired, although the temperature never reached a level that would have been harmful. The source of the problem was that the digital-to-analog converter used to convert the commanded temperature set point was physically only twelve bits wide, while the documentation and software on the ground specified sixteen bits. The effect was to silently truncate (i.e. set to zero) the most significant four bits of the commanded value.

This had never been a problem during the more than five years of the prime cryogenic mission since we didn't need to use those four truncated bits to set the desired temperature. However, we did need to set some of those bits to one to specify the desired temperature for the 
extended warm mission. To solve the anomaly, we patched the flight software to shift the offset and range of the digital-to-analog converter so that only the least-significant twelve bits were needed to specify the desired temperature set point.

The process of building, testing and approving the software patch took about one month. Once we were confident we had correctly diagnosed the problem, after about one week, we turned IRAC back on, let its temperature float and made some science observations. These images will require some extra, manual calibration to produce valid science data. During this time, we also decided to modify the sequencing processes we had originally designed for IWIC.

We made this decision in order to reduce the workload on the mission operations system teams. The decision also allowed us to be more efficient in the number of observations we could run on the observatory at the risk of a longer time to recover if we fell off the plan. And, we had to accept a longer cycle time between receipt of downlink and the next time when a sequence modified as a result of that data could be executed on board the observatory.

The original plan called for the mission operations teams to work two shifts per day for most of the duration of IWIC. We felt it they could not effectively maintain that schedule for a full extra month. In order to change to prime work shift only, we combined the two twelve-hour mini sequences per day into a single twenty-four-hour mini sequence. This allowed the mission operations teams to complete their work during prime shift, although they still worked seven

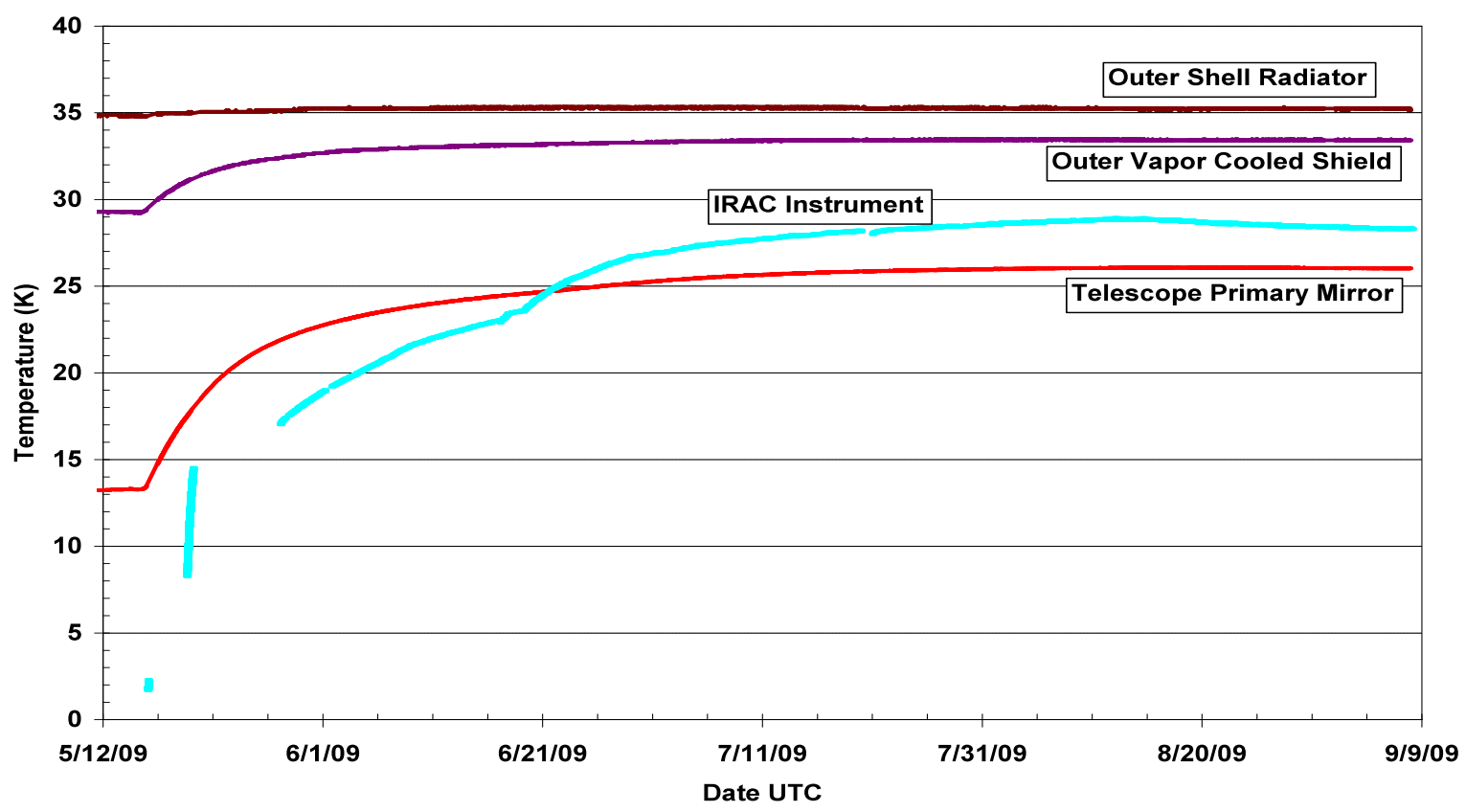

Figure 2. Spitzer's Temperature Profile During IWIC.

days per week. Some of the teams at the Spitzer Science Center had to retain their original schedule of two shifts per day or longer. The main effect of increasing the execution duration of the mini sequences to twenty-four hours was to delay receipt of data from the first half of the mini sequence by twelve hours. This delayed the time for modifying a subsequent sequence by twelve to twenty four hours. So, we had a reduced capability to respond quickly to changes in 
plan driven by results of a previous mini sequence. As a precaution, the first six mini sequences were built to be twelve hours long. And, we got extended telecommunications coverage so we could observe the beginning of the first mini sequence to observe the first use of the patched flight software.

After the end of IWIC, we discovered that the array temperature, initially set to $31 \mathrm{~K}$, could be reduced to $28.7 \mathrm{~K}$, as measured by the IRAC Cernox sensors. This allowed a reduction in the array bias voltages and a significant improvement in performance. So, we let the instrument cool back down slightly starting in mid-August. See Figure 2. This was followed in mid-September by a short recalibration of the instrument, and a change to some of the operating parameters.

\section{Conclusions}

The IWIC phase of the mission was slated for completed within six weeks of helium depletion in order to return to science operations. By modifying the IWIC planning and execution after the one-month delay due to the IRAC firmware anomaly, this goal was accomplished. We completed IWIC within the six weeks and accomplished all objectives. There were no further anomalies or large disruptions to the plan. We never had to idle the observatory because of the twenty-four-hour mini sequences. And, the longer sequences did prove to be more efficient. We had little idle time with each mini sequence and we got to use an extra hour or more per day observing that would have previously been devoted to a telecommunication pass. Had we kept the twelve-hour mini sequences, IWIC would have taken more calendar time to complete.

Table 3. Planned vs. Actual by Mission Phase

\begin{tabular}{|l|l|l|l|l|l|}
\hline $\begin{array}{l}\text { Mission } \\
\text { Phase }\end{array}$ & $\begin{array}{l}\text { Prime } \\
\text { Cryogenic }\end{array}$ & $\begin{array}{l}\text { Planned } \\
\text { Standby } \\
\text { Mode }\end{array}$ & IWIC Overall & $\begin{array}{l}\text { IWIC Post- } \\
\text { Anomaly } \\
\text { Only }\end{array}$ & $\begin{array}{l}\text { Extended } \\
\text { Warm }\end{array}$ \\
\hline $\begin{array}{l}\text { Expected } \\
\text { Total Duration }\end{array}$ & 5 years & 3 days & 42 days & 42 days & 2 years \\
\hline $\begin{array}{l}\text { Margin within } \\
\text { Total }\end{array}$ & $\mathrm{n} / \mathrm{a}$ & 1 day (33\%) & 8.4 days $(20 \%)$ & $\begin{array}{l}8.4 \text { days } \\
(20 \%)\end{array}$ & n/a \\
\hline Actual & 5.5 years & 3 days & 71 days & 39 days & n/a \\
\hline $\begin{array}{l}\text { Actual Margin } \\
\text { within Total }\end{array}$ & $\mathrm{n} / \mathrm{a}$ & 1 day $(33 \%)$ & $\begin{array}{l}-30 \text { days }(-71 \%) \\
\text { (Anomaly } \\
\text { recovery) }\end{array}$ & $\begin{array}{l}3 \text { days } \\
(7.1 \%)\end{array}$ & n/a \\
\hline
\end{tabular}

There are two ways, then, to summarize our overall performance, see Table 3 . If we look at the total duration, including the time to recover from the anomaly (33 days), IWIC took 72 days to complete and was 30 days, or $71 \%$ over the allocation. However, a more realistic measure is to consider only the time after the anomaly. By that measure, IWIC took 39 days to complete and was 3 days, or $7.1 \%$ under the allocation.

The planning strategy process to provide an early briefing to the MOS ensured that all teams were knowledgeable of the differences and expectations of the mission phases and transitions. The MOS staffing proved to be adequate to respond to the anomaly and re-planning effort. Also by adding the IWIC lead position the pace of staying on plan could be monitored for early detection of schedule compromise. The operating ground system maintained its fidelity and functionality throughout the entire IWIC with no impacts. An MOS calendar tool that was 
developed for the cryogenic mission was used for planning the sequences development through execution and provided accountability to track the IWIC objectives.

The frame table accuracy and focus was assessed continually during the IWIC. This assessment was made again once the temperature of the telescope reached equilibrium. No frame table update or focus adjustment was required at the completion of IWIC.

It was determined that the performance of the IRAC instrument could be significantly improved by modifying both the operating temperature and the array bias requiring both a new science block library and about two hundred hours of IRAC calibrations which were carried out about ten weeks into the nominal warm mission.

\section{Acknowledgments}

The research described in this paper was carried out at the Jet Propulsion Laboratory, California Institute of Technology, under a contract with the National Aeronautics and Space Administration.

The authors would like to thank the following people who helped us along the way, in alphabetical order: Sean Carey, Suzanne Dodd, Paul Finley, Mark Fisher, Bolinda Kahr, Charles Scott, Lisa Storrie-Lombardi and Robert K. Wilson.

\section{References}

\footnotetext{
${ }^{1}$ Bennett, et al., "The Spitzer Science Center System Description and Lessons Learned Due to Two Years of Operations," Proc. SPIE 6270, Bellingham, WA, USA, (2006).

${ }^{2}$ Dodd, Levine, "The Spitzer Space Telescope's Performance: Getting the most out of a Great Observatory," Proc. SPIE 6270, Bellingham, WA, USA, (2006).

${ }^{3}$ Dodd, Storrie-Lombardi, Scott, "Spitzer's Model for Dealing with the End of the Cryogenic Mission," Proceedings of the SPIE - Astronomical Instrumentation Conference, Vol. 7016, SPIE, Bellingham, WA, USA, (2008).

${ }^{4}$ Mahoney, et al., "Spitzer Scheduling Challenges: Cold and Warm," Proceedings of the SPIE - Astronomical Instrumentation Conference, Vol. 7016, SPIE, Bellingham, WA, USA, (2008).

${ }^{5}$ Sarrel, Hunt, "Evaluating Requirements on the Spitzer Mission Operations System Based on Flight Operations Experience," Proceedings of the SPIE - Astronomical Instrumentation Conference, Vol. 7016, SPIE, Bellingham, WA, USA, (2006).

${ }^{6}$ Scott, et al., "Spitzer observatory operations - increasing efficiency in mission operations," Proc. SPIE 6270, Bellingham, WA, USA, (2006).

${ }^{7}$ Scott, Wilson, "Spitzer pre launch mission operations system - the road to launch," Proc. SPIE 6270, Bellingham, WA, USA, (2006).

${ }^{8}$ Wilson, Scott, "NASA's Spitzer Space Telescope's Operational Mission Experience," Proc. SPIE 6270, Bellingham, WA, USA, (2006).
} 